\title{
Comparison of short-term clinical outcomes of manipulation under anesthesia and arthroscopic capsular release in treating frozen shoulder
}

Enes ULUYARDIMCI' ${ }^{1}$ Durmus Ali OCGUDER²

\author{
1 Department of Orthopaedics and Traumatology, Develi Hatice-Muammer Kocaturk State Hospital, Kayseri, Turkey \\ ${ }^{2}$ Department of Orthopaedics and Traumatology, Ankara City Hospital, Ankara, Turkey \\ Correspondence \\ Enes ULUYARDIMCI \\ Develi Hatice-Muammer Kocatürk Devlet Hastanesi, Ortopedi ve Travmatoloji Bölümü, Kayseri, Türkiye \\ e-mail: enesuluyardmc7@hotmail.com
}

\section{ABSTRACT}

Manipulation under anesthesia (MUA) and arthroscopic capsular release (ACR) are the two most common treatment options in the surgical treatment of refractory frozen shoulder (FS). This study aimed to compare the short-term clinical outcomes of MUA and ACR in refractory FS treatment.

This retrospective study included 17 patients who received MUA and 15 patients who received ACR due to refractory FS. The patients were evaluated clinically and functionally with the range of motion (ROM), American Shoulder and Elbow Surgeons (ASES) score, and visual analogue scale (VAS) pain score.

The mean follow-up time was $17.3 \pm 9.8$ months and $16.9 \pm 9.1$ months in the MUA and ACR groups, respectively, with no statistically significant difference between the two groups $(P=0.882)$. The mean age was $56.1 \pm 6.8$ and $55.7 \pm 7.2$ in the MUA and ACR groups, respectively, with no statistically significant difference between the two groups $(P=0.882)$. The two groups were found to be similar in terms of sex, affected side, duration of symptoms, presence of diabetes, and presence of thyroid disease $(P>0.970)$. In both groups, forward flexion, external rotation, and internal rotation improved significantly compared with preoperative values $(P<0.001)$. No significant difference was found between the two groups in terms of ROM, ASES score, and VAS pain score at the last follow-ups $(P>0.576)$.

Successful clinical and functional outcomes can be obtained with both MUA and ACR in the surgical treatment of refractory FS without major serious complications. In the present study, no significant difference was found between the MUA and ACR groups.

Key words: Arthroscopy, capsular release, frozen shoulder, manipulation

\section{INTRODUCTION}

A clinical condition characterized by chronic pain and progressive range of motion (ROM) deficits in the shoulder joint is traditionally known as adhesive capsulitis (1). Some authors defined this situation as stiff shoulder and called primary idiopathic stiff shoulder as "frozen shoulder" (2). Although predisposing factors such as diabetes, thyroid diseases, and smoking may exist, the cause of frozen shoulder (FS) is unknown and is not secondary to trauma or a specific shoulder disease (2). In addition, no significant pathology other than the possible presence of osteopenia or calcific tendinitis is seen in radiographic evaluation (3).
FS is observed at a rate of about $2 \%-5 \%$ in the general population and generally affects women aged $40-60$ years more frequently (4-7). The natural course of FS consists of three phases: freezing, frozen, and thawing, and can last up to 2-3 years (8). Although FS is a self-limiting pathology, chronic pain or stiffness may persist in about half of the patients, and full recovery may not be achieved $(8,9)$. Although no consensus exists in the literature regarding its treatment, oral medication, intra-articular injection, physical therapy, joint distension, and high-frequency ultrasound constitute nonoperative treatment methods $(1,2)$. Success can be achieved with nonoperative 
treatment in the majority of patients, but manipulation under anesthesia (MUA) or arthroscopic capsular release (ACR) is recommended in refractory cases despite at least 6 months of nonoperative treatment $(7,10)$

MUA is a well-known and effective treatment option for refractory FS (11). However, some authors reported no significant difference in the clinical outcomes between MUA and other treatment options $(12,13)$. In addition, the complications of MUA such as proximal humerus fracture, rotator cuff tear, and brachial plexus palsy are known (10). Thanks to the rapid development of arthroscopic techniques, arthroscopic treatment of FS has become popular in the last decade. Faster recovery can be achieved with ACR compared with other treatment methods, and mid-term and long-term successful outcomes have been reported in the treatment of refractory FS with ACR $(14,15)$.

The present study was aimed to compare the short-term clinical outcomes of MUA and ACR in refractory FS treatment. It was hypothesized that ACR was associated with better clinical outcomes compared with MUA.

\section{MATERIAL AND METHODS}

In this retrospective clinical study, patients who underwent surgical treatment during 2014-2017 for refractory FS were evaluated. The study data were retrieved from the archiving system consisting of patient files during the hospitalization, and follow-up cards were used in the outpatient clinic. The inclusion criteria were defined as patients with refractory FS having a functional restriction of both active and passive shoulder motion and in whom no intrinsic or extrinsic shoulder pathology other than osteopenia was detected on the radiological examination of the glenohumeral joint. Refractory FS was defined as recalcitrant to nonoperative management (oral medications, physical therapy, and injections) for 6 months and cases with restriction in the shoulder ROM compared with the contralateral side. Patients with rotator cuff tear, calcific tendinitis, shoulder osteoarthritis, posttraumatic or postsurgical etiology, incomplete patient records, and less than 6 months of follow-up, and patients whose shoulder functions were affected due to a musculoskeletal or neurological disease were excluded from the study. MUA or ACR is applied as surgical treatment in refractory FS cases in the clinic. The patients who received MUA formed the MUA group, and the patients received ACR formed the ACR group. Thirty-two patients met the selection criteria and were included in the study. The MUA group comprised 17 patients, while the ACR group comprised 15 patients. Written informed consent was obtained from each patient. The study was conducted in accordance with the principles of the Declaration of Helsinki.

MUA was applied under propofol anesthesia with manual mask ventilation while the patient was in the supine position. Preoperative ROM measurements were conducted after anesthesia before the procedure. The scapula was fixed posteriorly, and forward flexion was applied gently first. Then, gradual abduction was performed while the shoulder was supported by an assistant. Finally, external and internal rotation was realized. Following the manipulation, intra-articular injection (40 mg methylprednisolone, $5 \mathrm{~mL}$ of lidocaine, and $5 \mathrm{~mL}$ of saline solution) was applied. ACR was performed in the beach chair position under general anesthesia. In the ACR group, preoperative ROM measurements were conducted after anesthesia before the procedure. Primarily, the posterior portal was used for imaging. After the diagnostic arthroscopy, the anterior release was applied with a radiofrequency device. The capsular release was continued from the anterosuperior to the inferior side (6 o'clock position). An arthroscopic basket punch was used to avoid iatrogenic axillar nerve damage on the inferior side. Then, a posterior capsular release was performed using the anterior portal for imaging. Then, bursectomy was performed, and subacromial adhesions were released after passing the subacromial area. Finally, after the $360^{\circ}$ capsular release, the ROM was controlled by applying forward flexion, abduction, external rotation, and internal rotation. All patients were given analgesics and kept on an arm sling for pain control. Passive ROM exercises were started on the first postoperative day. The same rehabilitation protocol was used in both groups.

Age, sex, affected side, history of diabetes or thyroid disease, duration of preoperative symptoms, 
and preoperative ROM were recorded. Patients were evaluated with ROM, American Shoulder and Elbow Surgeons (ASES) score (16), and visual analogue scale (VAS) pain score at the last follow-up. In the VAS pain score, 0 indicated "no pain" and 10 indicated "severe pain." Vertebrae were enumerated for the quantitative analysis of internal rotation. Accordingly, between T1 and T12 19-8, and L1 to L5 7-3, sacrum was numbered 2 and buttock was numbered 1. Preoperative ASES and VAS pain scores were not available. Complications and reoperations were recorded.

\section{Statistical analyses}

The SPSS 25.0 software package was used in the statistical analysis of the data. Categorical measurements were expressed in numbers and percentages, and continuous measurements were expressed in mean and standard deviation values. The chi-square test or Fisher's test was used in the comparison of categorical variables. Distributions were analyzed for a comparison of continuous measurements between the groups. The Student $t$ test was used for variables with a parametric distribution and the Mann-Whitney $\mathrm{U}$ test for variables with a nonparametric distribution. $P<0.05$ indicated a statistically significant difference in all tests.

\section{RESULTS}

The mean age was $56.1 \pm 6.8$ and $55.7 \pm 7.2$ in the MUA and ACR groups, respectively, with no statistically significant difference between the two groups $(P=0.882)$. The two groups were found to be similar in terms of sex, affected side, duration of symptoms, presence of diabetes, and presence of thyroid disease (Table 1). The mean follow-up time was $17.3 \pm 9.8$ months and $16.9 \pm 9.1$ months in the MUA and ACR groups, respectively, with no statistically significant difference between the two groups $(P=0.882)$. In both groups, forward flexion, external rotation, and internal rotation improved significantly compared with preoperative values $(P<0.001)$ (Table 2$)$. At the final

Table 1 Preoperative patient demographics

\begin{tabular}{llll}
\hline & $\begin{array}{l}\text { MUA group } \\
(n=17)\end{array}$ & $\begin{array}{l}\text { ACR group } \\
(n=15)\end{array}$ & $P$ value \\
\hline $\begin{array}{l}\text { Age, mean } \pm \text { SD, year } \\
\text { Sex }\end{array}$ & $56.1 \pm 6.8$ & $55.7 \pm 7.2$ & 0.882 \\
$\quad$ Female/Male patients, $n$ (\%) & $13 / 4(76.5 \%)$ & $11 / 4(73.3 \%)$ & 1.000 \\
Affected side & & & 1.000 \\
$\quad$ Right/Left, $n$ & $12 / 5$ & $10 / 5$ & 0.970 \\
Duration of symptoms, mean \pm SD, month & $13.6 \pm 4.3$ & $14.1 \pm 6.2$ & 1.000 \\
History of diabetes, $n$ & 6 & 6 & 1.000 \\
History of thyroid disease, $n$ & 2 & 1 & \\
\hline
\end{tabular}

ACR, Arthroscopic capsular release; MUA, manipulation under anesthesia; SD, standard deviation.

Table 2 Preoperative and postoperative range of motion

\begin{tabular}{lllllll}
\hline & \multicolumn{2}{c}{ Forward flexion } & \multicolumn{2}{c}{ External rotation } & \multicolumn{2}{c}{ Internal rotation } \\
& Preoperative & Postoperative & Preoperative & Postoperative & Preoperative & Postoperative \\
\hline MUA & $105.3 \pm 11.4$ & $157.5 \pm 19.3$ & $24.7 \pm 9.1$ & $51.5 \pm 13.4$ & $3.6 \pm 2.0$ & $10.3 \pm 2.1$ \\
ACR & $104.7 \pm 14.3$ & $158.6 \pm 20.9$ & $24.3 \pm 9.6$ & $52.3 \pm 13.1$ & $3.3 \pm 1.9$ & $10.6 \pm 2.3$ \\
P & 0.941 & 0.682 & 0.911 & 0.911 & 0.710 & 0.576
\end{tabular}


Table 3 Postoperative outcomes

\begin{tabular}{llll}
\hline & $\begin{array}{l}\text { MUA group } \\
(n=17)\end{array}$ & $\begin{array}{l}\text { ACR group } \\
(n=15)\end{array}$ & $P$ value \\
\hline Follow-up period, month & $17.3 \pm 9.8$ & $16.9 \pm 9.1$ & 0.882 \\
ASES score, points & $91.6 \pm 4.9$ & $92.1 \pm 5.8$ & 0.852 \\
VAS pain score, points & $0.47 \pm 07$ & $0.40 \pm 0.7$ & 0.737 \\
\hline
\end{tabular}

Values are expressed as mean $\pm \mathrm{SD}$.

ACR: Arthroscopic capsular release, ASES: American Shoulder and Elbow Surgeons, MUA: manipulation under anesthesia,

SD: standard deviation, VAS: visual analogue scale.

follow-up visits, the mean forward flexion, external rotation, and internal rotation values were similar in the MUA and ACR groups $(P>0.576)$. At the last follow-up, the ASES score was $91.6 \pm 4.9$ points and $92.1 \pm 5.8$ points in the MUA and ACR groups, respectively, with no significant difference between the two groups ( $P$ $=0.852$ ). At the last follow-up, the VAS pain score was $0.47 \pm 0.7$ and $0.40 \pm 0.7$ in the MUA and ACR groups, respectively, and the two groups were found to be similar $(P=0.737$ ) (Table 3). One patient in the MUA group developed recurrent pain in the second postoperative month. Early recurrent stiffness developed in one patient in the ACR group in the third postoperative week. In the last follow-up of both patients, satisfactory outcomes were obtained in terms of clinical scores and ROM. No revision surgeries and no serious complications such as infection, fracture, instability, and neurovascular injury were reported.

\section{DISCUSSION}

The main finding of the present study was that MUA and ACR were similar in clinical scores, pain, and ROM in refractory FS. Farrel et al. reported the mean forward flexion of the 19 shoulders of the 18 patients treated with MUA as $168^{\circ}$ and external rotation as $67^{\circ}$ after an average of 15 years of follow-up (11). After 12 years of follow-up, Kim et al. compared MUA and ACR and found that the mean forward flexion, external rotation, and ASES scores in the MUA group was $167.6^{\circ} \pm$ $5.2^{\circ}, 73.0^{\circ} \pm 9.3^{\circ}$, and $89.1 \pm 14.4$, respectively (17). In the same study, these values were $166.3^{\circ} \pm 7.0^{\circ}, 61.3^{\circ}$ $\pm 10.2^{\circ}$, and $84.7 \pm 15.2$, respectively, in the ACR group. Cvetanovich et al. reported the average forward flexion, external rotation, and ASES scores as $156.2^{\circ} \pm$ $16.1^{\circ}, 56.8^{\circ} \pm 15.7^{\circ}$, and $97.0 \pm 4.7$, respectively, as a result of the $360^{\circ}$ ACR they applied in the lateral decubitus position with at least 2-year follow-up (14). Hence, the results of the present study were consistent with published findings.

Surgical treatment is recommended in refractory cases where nonoperative management results in at least 6 months due to the natural history of FS and the high success rate of nonoperative treatment (7). Which surgical treatment should be chosen is still controversial; MUA and ACR are the two most preferred treatment options $(2,10)$. Successful results of MUA, which is a well-known treatment, have been published in the short and long term; it has been emphasized that a rapid improvement can be achieved in ROM and functional outcomes with MUA $(11,17,18)$. On the contrary, some authors reported no significant difference in terms of clinical outcomes between MUA and other treatment options (12,13). Kvimaki et al. showed that MUA and home exercise programs were related to similar outcomes and concluded that MUA was not superior to a home exercise program (13).

Successful results have been published in recent years after the widespread use of arthroscopic techniques in the treatment of FS. Barnes et al. achieved significant and rapid improvements in ROM, pain, and shoulder functions starting from the first postoperative week with $A C R$, and reported that this improvement continued in 6, 12, and 24 weeks (19). Cvetanovich et al. concluded that $360^{\circ} \mathrm{ACR}$ in FS treatment provided early and lasting improvement in ROM and excellent functional results with low complication and revision rates (14). 
MUA is actually an uncontrolled release associated with potential complications such as fractures, dislocation, and brachial plexus injury (10). Hemarthrosis, hemorrhage, and tearing of glenohumeral ligaments and capsule, rotator cuff tears, and labral tears are arthroscopically documented intra-articular effects of MUA $(21,21)$. ACR provides precise and controlled release of the capsule and ligaments, reducing the risk of traumatic complications that may occur due to the forces applied during manipulation. In addition, releasing with the radiofrequency device delays healing and prevents re-occurring adhesions (2). During inferior capsular release in $A C R$, care should be taken in terms of the risk of axillary nerve damage due to its anatomic location. Despite the potential disadvantages of MUA and the potential advantages of ACR, the superiority of ACR over MUA in terms of clinical results has not been proven yet. Kim et al. compared MUA and ACR in refractory FS treatment; VAS pain score, ASES score, and forward flexion were reported to be significantly better at a 3-month follow-up in the MUA group compared with the ACR group, while the 12-month follow-up result showed that both the groups were reported to be similar in terms of these results (17). Lee et al. reported similar MUA and ACR in terms of clinical and functional outcomes as a result of a 3-month follow-up (22). Similarly, in the present study, no significant difference was observed between MUA and ACR in refractory FS treatment. In this respect, the results of the present study were not compatible with the hypothesis. In addition, no serious major complication was observed in either group.

The present study had several limitations. Data of seven patients could not be included in the study due to its retrospective design. Preoperative data were similar between the two groups, although it was nonrandomized. Also, this study had small sample size. Prospective studies comparing ACR and MUA are limited in the literature.

\section{CONCLUSIONS}

Successful clinical and functional outcomes can be obtained with both MUA and ACR in the surgical treatment of refractory FS without major serious complications. In the present study, no significant differ- ence was found between MUA and ACR. Prospective, randomized, large-sample studies comparing MUA and ACR should be conducted in the future.

\section{REFERENCES}

1. Forsythe B, Lavoie-Gagne O, Patel BH, Lu Y, Ritz E, Chahla J, Okoroha KR, Allen AA, Nwachukwu BU. Efficacy of Arthroscopic Surgery in the Management of Adhesive Capsulitis: A Systematic Review and Network Meta-analysis of Randomized Controlled Trials. Arthroscopy. 2020 Nov 20:S0749-8063(20)30804-5. doi: 10.1016/j.arthro.2020.09.041. Epub ahead of print. PMID: 33221429.

2. Itoi $E$, Arce $G$, Bain Gl, Diercks RL, Guttmann D, Imhoff $A B$, Mazzocca AD, Sugaya H, Yoo YS. Shoulder Stiffness: Current Concepts and Concerns. Arthroscopy 2016;32:1402-14.

3. Zuckerman JD, Rokito A. Frozen shoulder: a consensus definition. J Shoulder Elbow Surg 2011;20:322-5.

4. Lee JH, Kim SB, Lee KW, Lee SJ, Lee JU. Effect of Hypertonic Saline in Intra-Articular Hydraulic Distension for Adhesive Capsulitis. PM R 2015;7:721-6.

5. Lewis J. Frozen shoulder contracture syndrome - Aetiology, diagnosis and management. Man Ther 2015;20:2-9.

6. Griggs SM, Ahn A, Green A. Idiopathic adhesive capsulitis: A prospective functional outcome study of nonoperative treatment. J Bone Joint Surg Am 2000;82:1398-407.

7. Hsu JE, Anakwenze OA, Warrender WJ, Abboud JA. Current review of adhesive capsulitis. J Shoulder Elbow Surg 2011;20:502-14.

8. Reeves B. The natural history of the frozen shoulder syndrome. Scand J Rheumatol 1975;4:193-6.

9. Hand C, Clipsham K Fau - Rees JL, Rees Jl Fau - Carr AJ, Carr AJ. Long-term outcome of frozen shoulder. Journal of Shoulder and Elbow Surgery 2008;17:231-6.

10. Grant JA, Schroeder N, Miller BS, Carpenter JE. Comparison of manipulation and arthroscopic capsular release for adhesive capsulitis: a systematic review. J Shoulder Elbow Surg 2013;22:1135-45.

11. Farrell CM, Sperling JW, Cofield RH. Manipulation for frozen shoulder: Long-term results. J Shoulder Elbow Surg 2005;14:480-4.

12. Maund E, Craig D, Suekarran S, et al. Management of frozen shoulder: A systematic review and costeffectiveness analysis. Health Technol Assess 2012;16:1-264.

13. Kivimaki J, Pohjolainen T, Malmivaara A, et al. Manipulation under anesthesia with home exercises versus home exercises alone in the treatment of frozen shoulder: A randomized, controlled trial with 125 patients. J Shoulder Elbow Surg 2007; $16: 722-6$

14. Cvetanovich GL, Leroux TS, Bernardoni ED, Hamamoto JT, Saltzman BM, Verma NN, Romeo AA. Clinical Outcomes of Arthroscopic $360^{\circ}$ Capsular Release for Idiopathic Adhesive Capsulitis in the Lateral Decubitus Position. Arthroscopy 2018;34:764-70.

15. Le Lievre HM, Murrell GA. Long-term outcomes after arthroscopic capsular release for idiopathic adhesive capsulitis. J Bone Joint Surg Am 2012;94:1208-16.

16. Michener LA, McClure PW, Sennett BJ. American Shoulder and Elbow Surgeons Standardized Shoulder Assessment Form, patient self-report section: reliability, validity, and responsiveness. J Shoulder Elbow Surg 2002;11:587-94. 
17. Kim DH, Song KS, Min BW, Bae KC, Lim YJ, Cho CH. Early Clinical Outcomes of Manipulation under Anesthesia for Refractory Adhesive Capsulitis: Comparison with Arthroscopic Capsular Release. Clin Orthop Surg 2020;12:217-23.

18. Dodenhoff RM, Levy O, Wilson A, Copeland SA. Manipulation under anesthesia for primary frozen shoulder: effect on early recovery and return to activity. J Shoulder Elbow Surg 2000;9:23-6.

19. Barnes CP, Lam PH, Murrell GA. Short-term outcomes after arthroscopic capsular release for adhesive capsulitis. J Shoulder Elbow Surg 2016;25:e256-64. doi: 10.1016/j.jse.2015.12.025. Epub 2016 Mar 8.
20. Loew M, Heichel TO, Lehner B. Intraarticular lesions in primary frozen shoulder after manipulation under general anesthesia. J Shoulder Elbow Surg 2005;14:16-21.

21. Uitvlugt G, Detrisac DA, Johnson LL, Austin MD, Johnson C. Arthroscopic observations before and after manipulation of frozen shoulder. Arthroscopy 1993;9:181-5.

22. Lee SJ, Jang JH, Hyun YS. Can manipulation under anesthesia alone provide clinical outcomes similar to arthroscopic circumferential capsular release in primary frozen shoulder (FS)?: the necessity of arthroscopic capsular release in primary FS. Clin Shoulder Elb 2020;23:169-77. 